\title{
Competition between electronic energy transfer and relaxation in Xe-doped Ar and Ne matrices studied by photoelectron spectroscopy*
}

\author{
N. Schwentner \\ Christian Albrechts Universität, 23 Kiel, Germany \\ E. E. Koch \\ Deutsches Elektronen-Synchrotron DESY, 2 Hamburg 52, Germany \\ (Received 15 January 1976)
}

\begin{abstract}
Thin films of solid Ar and Ne doped with 1-at.\% Xe were excited with photons in the energy range from 10 to $20 \mathrm{eV}$ in order to measure the energy distribution of the emitted electrons. Binding energies of the host and guest levels are deduced. When host excitons are excited, strong emission of electrons is observed indicating an efficient transfer of the host exciton energy to the Xe guest atoms. The energy of the free excitons is transferred as can be deduced from the kinetic energy of the photoemitted electrons rather than the energy of the bound (self-trapped) excitons which are observed in luminescence experiments. Furthermore, there is a striking difference between the Ar and Ne matrix: In the Ne matrix a fast relaxation from the $n=2$ to the $n=1$ state was observed and only the energy of the $n=1$ exciton is transferred even when higher excitons are excited, in contrast to Ar, where the transferred energy is higher for excitation of the $n=2$ excitons than for $n=1$. From these observations time hierarchies for the competition between electronic energy transfer and relaxation are deduced.
\end{abstract}

\section{INTRODUCTION}

Excitation of insulators by light, $\mathrm{x}$ rays, $\gamma$ particles, protons, electrons, or $\alpha$ particles leads to the emission of light which is characteristic for the sample but rather independent of the special source. The dissipation of the excitation energy up to the point of luminescence has attracted increasing attention which is indicated by the large amount of recent contributions dealing with problems of radiationless transitions. ${ }^{1}$ In the present study photoelectron energy-distribution measurements, where the energy of the exciting light could be varied, have been used to investigate such decay processes.

During the last decade, the optical properties of fundamental insulators, viz., the rare-gas solids, ${ }^{2}$ and their luminescence spectra ${ }^{3-6}$ have been studied. The absorption spectra are dominated by exciton series which converge in a hydrogenic fashion to the band gap. The energies of the luminescence bands are smaller than the lowest absorption line and the emission is attributed to trapped excitons, i.e., the decay of vibrationally relaxed, electronically excited homonuclear raregas diatomic molecules. ${ }^{7}$ (For the special case of solid Ne see Ref. 37.) Besides theoretical interest, the efforts to develop more efficient vuv laser $\mathrm{s}^{8}$ stimulate the study of the involved decay channels, including a special radiationless transition involving the energy transfer of host excitation energy to guest atoms in doped rare-gas solids. ${ }^{9}$ From theoretical estimates ${ }^{10,11}$ and from the results of recent photoelectron yield studies, ${ }^{12}$ it is expected that many relaxation processes are fast, with time constants in the $10^{-11}$ - to $10^{-13}-\mathrm{sec}$ range. By application of ultrashort light pulses from mode-locked lasers, decay measurements in the picosecond time scale have been carried out. ${ }^{13}$ Such light sources are not available for the high photon energies ( $\hbar \omega>8 \mathrm{eV}$ ) which are required for the large band gaps of the rare-gas solids. Thus one is restricted to less-direct experiments.

In recent luminescence experiments, efforts have been made to obtain more information about these states by the use of monochromatic radiation. ${ }^{5}$ By these means, different states can be separately populated. However, the resulting light emission is mainly observed from the lowest states.

Thus new experiments are required in order to determine both the energies of the upper states and the time hierarchy involved.

From the energy distribution curves (EDC's) of photoelectrons, the structure of the valence bands and the lowest conduction bands of all the raregas solids has been deduced. ${ }^{14}$ For highly excited electrons with kinetic energies exceeding the band gap $E_{G}$ (excitation energy $\geq 2 E_{G}$ ) the scattering length for electron-electron inelastic scattering falls from $\approx 1000 \AA$ to below ${ }^{15} 10 \AA$, i.e., a time constant for this process shorter than $\approx 10^{-15} \mathrm{sec}$. The kinetic energy of low-energy electrons in the conduction band is dissipated only by interaction with the lattice (phonons, defects) because inelastic electron-electron scattering is forbidden by the band gap. ${ }^{15,16}$ 

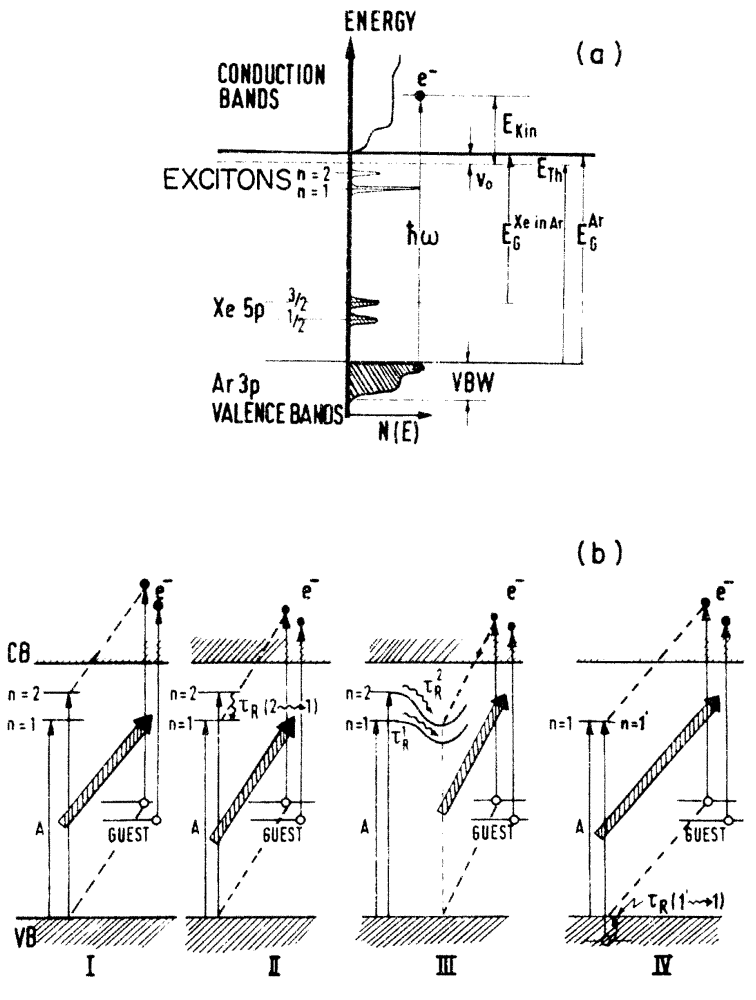

FIG. 1. (a) Schematic scheme of the energy levels involved in photoelectron emission from doped solid rare gases depicted for the case Xe in Ar. The energies given are discussed in the text; VBW denotes the valence bandwidth. (b) Schematic scheme for various energytransfer and relaxation processes discussed in the text. Case I, energy transfer of free excitons to guest atoms; case II, relaxation of the free $n=2$ to the $n=1$ exciton state and subsequent energy transfer; case III, relaxation of the exciton states to trapped exciton states and subsequent energy transfer; case IV, relaxation of the hole in the host valence band and subsequent energy transfer, this process has also to be considered for cases I, II and III.

In this paper we are mainly concerned with relaxation processes in the excitonic region below the bottom of the conduction band. The exciton series observed in optical spectra of pure and doped rare-gas solids can be satisfactorily interpreted in terms of Wannier series converging to the bottom of the conduction band for the members $n \geq 2 .^{3}$ The lowest exciton $(n=1)$ is of the intermediate type (between Wannier and Frenkel) but can be described by a $n=1$ Wannier state subjected to a large central-cell correction. ${ }^{3}$ For $\mathrm{Ar}, \mathrm{Kr}$, and $\mathrm{Xe}$ and the impurity states of these elements two series split by the spin-orbit coupling in the $n p$-valence shells are exhibited. They are denoted by $n\left(j=\frac{3}{2}\right)$ and $n^{\prime}\left(j=\frac{1}{2}\right)$ (see Fig. 1 ). The $n=1$ and $n=2$ exciton states lie below the vac- uum level and do not contribute to photoelectron emission directly. ${ }^{2,17,18}$ Recent photoelectron yield measurements of pure and doped Ar and $\mathrm{Ne}$ showed that these excitons decay and excite electrons above the vacuum level via energy transfer to guest atoms or to the gold substrate. ${ }^{12,19}$ From the knowledge of the incident-photon energy and the observed kinetic energies of the electrons in the EDC's the relaxation energy before energy transfer can be deduced. Thus we can compare the relaxation time to different states with the time constant for energy transfer. For example, when the $n=2$ exciton of the host is excited a competition between relaxation to the $n=1$ exciton, relaxation to the self-trapped exciton, and energy transfer to guest atoms is expected. For Ar and Ne, theoretical calculations for both relaxation time constants are available. ${ }^{10,11}$ The difference in binding energies of $n=1$ and $n=2$ states in Ar as well as in $\mathrm{Ne}$ exceeds $1 \mathrm{eV}$ and can be well resolved with the resolution of our electron analyzer of $0.2 \mathrm{eV}$. The impurity states of Xe lie high enough above the host valence band to be ionized even by the $n=1$ exciton of the host. The various possible processes are sketched in Fig. 1(b).

After a short description of the experimental arrangement (Sec. II) EDC's for 1-at.\% Xe in Ar and 1 -at. \% Xe in Ne matrix are presented for several photon energies (Sec. III). The energies involved are discussed in Sec. IV while the time hierarchy is deduced in Sec. V.

\section{EXPERIMENTAL PROCEDURE}

The synchrotron radiation of the Deutsches Elektronen-Synchrotron (DESY) together with a normal-incidence monochromator (resolution $2 \AA$ ) served as a light source for photon energies from 5 to $30 \mathrm{eV}$ with a photon flux at the sample of typically $10^{9}$ photons $/ \mathrm{sec}^{20,21}$ Attached to the UHV sample chamber (typical pressure $1 \times 10^{-10}$ Torr) were (i) a bakeable liquid-He cryostat, (ii) an electron energy analyzer, (iii) a turnable open photomultiplier to measure the sample reflectance, and (iv) a UHV gas-handling system (Fig. 2).

The incident light beam hits the sample with an angle of incidence of $45^{\circ}$, illuminating an area of $10 \mathrm{~mm}^{2}$. A gold film served as a substrate which was isolated from the cryostat by a quartz disk. The photoelectrons were preaccelerated by the applied bias voltage $V_{p}$ to $5 \mathrm{~V}$. The electron energy analyzer is mounted normal to the sample surface and accepts electrons within a cone of $3^{\circ}$. The electrons are selected according to their energy by a combination of a retarding grid and electrostatic lenses. Counting rates of 1000 counts/ sec were typical. For more details see Ref. 22. 


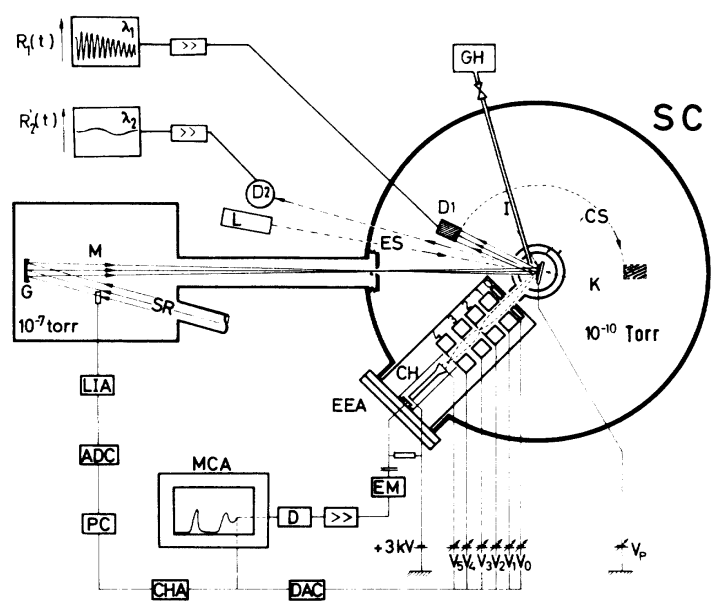

FIG. 2. Setup for simultaneous reflection and photoemission experiments. Synchrotron light (SR) enters the sample chamber (SC) from the monochromator (M) with concave grating $(G)$ via the exit slit (ES). A cryostat (K) with two cryoshields (CS) and an insulated sample substrate (I), an open electrostatic photomultiplier (D1), a gas-handling system (GH), and a photoelectron energy analyzer (EEA) with a channeltron as detector $(\mathrm{CH})$ are incorporated into the sample chamber. Photoelectron analysis: $V_{0}$ to $V_{5}$, lens voltages; $V_{p}$, sample voltage; EM, emitter follower; D. discriminator; MCA, multichannel analyzer; DAC, digital-analog converter. Channel advance is triggered by a reference signal via lockin amplifier (LIA), analog-digital converter (ADC), and a preset counter (PC). The reflectance as a function of wavelength is measured by D1. Film thickness is determined by comparing the reflectance $R_{1}(t)$ at $\lambda_{1}$ (vuv) and $R_{2}(t)$ at $\lambda_{2}$ [laser wavelength, laser (L) via detector D2] simultaneously during evaporation time $t$.

Photoelectron-emission measurements on raregas solids are hampered by strong charging effects. ${ }^{17(a), 23}$ Sample charging was minimized by the preparation of thin films with thicknesses of the order of $50 \AA$. The growth of the films was monitored during deposition of the gas by measuring continuously the oscillations in the reflectance in the transparent region of the material (Ar, $1100 \AA$; $\mathrm{Ne}, 800 \AA$ ). For the calculation of the film thickness, the formulas of Ref. 24 were applied to the solid-rare-gas-Au sandwich with optical constants taken from the literature. ${ }^{25,26}$ Further the illumination time was held to a minimum (1-5 min per spectrum) to avoid accumulation of charge. Consequently the statistics were not always as good as might be desired. In the spectra presented charging was less than $0.3 \mathrm{eV}$.

For the preparation of the films Matheson research-grade gases with a purity of better than $99.997 \%$ for Xe, $99.9999 \%$ for Ar, and $99.995 \%$ for Ne were used. The given doping concentrations correspond to the ratio of the partial pressures of the constituents in the gas-handling system (total pressure 1000 Torr). The Xe-Ne mixtures were frozen at substrate temperatures of $6 \mathrm{~K}$, the XeAr mixtures at $15 \mathrm{~K}$. No attempts for annealing were made.

\section{RESULTS}

\section{A. $\mathrm{Xe}$ in $\mathrm{Ar}$}

In the right-hand part of Fig. 3 EDC's of a 50 - $\AA$ thick film of 1-at.\% Xe in Ar are presented for several photon energies as a parameter. In order to show clearly the structure within each EDC the counting rates for each spectrum are arbitrarily normalized. For a quantitative comparison the total areas of the EDC's normalized to the same incident-light intensity are given by the crosses in the left-hand part of Fig. 3. They are compared with the yield curve of a 60 - $\AA$-thick film of 1 -at.\%

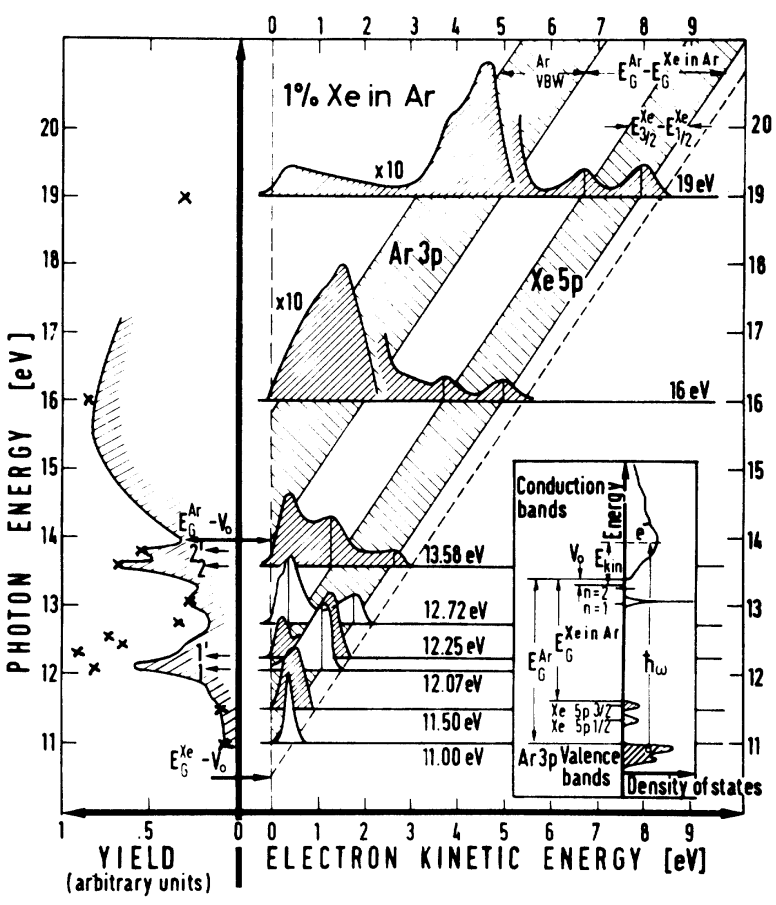

FIG. 3. Right part (EDC's): Photoelectron energydistribution curves (counting rates versus kinetic energy) of 1-at.\% Xe in Ar for a spectrum of photon energies. The film thickness was $50 \AA$. For normalization and subtraction of background see text. For convenience the relevant energy levels are shown in the insert. Left part (yield): The crosses represent the total number of emitted electrons from the EDC's. For comparison the yield of 1-at.\% Xe at Ar (Ref. 12) of a $60-\AA$-thick film is shown (solid line). The two sets of data were adjusted at $\hbar \omega=11$ and $11.5 \mathrm{eV}$ (gold substrate). The energies of the $n=1,1^{\prime}, 2$, and $2^{\prime}$ exciton states are marked. 
Xe in Ar as determined in Ref. 12. Here and in Fig. 4 a structureless background caused by hot electrons from the gold substrate was subtracted from the yield and EDC spectra. For $\hbar \omega=16$ and $19 \mathrm{eV}$ (Fig. 3) this background was not subtracted in order to show its contribution to the EDC's.

From Fig. 3 the common features of the yield curve and the total areas of the EDC's of a strong enhancement in (i) the region of the $n=1$ and $n=2$ excitons and (ii) in the region of interband transitions are immediately evident. However, quantitative agreement cannot be expected because of the different angle of incidence of the light. Furthermore, in the EDC's only electrons within a cone of $3^{\circ}$ are accepted, whereas in the yield spectra all the emitted electrons are collected.

In the EDC's the zero kinetic energy corresponds to the vacuum level of the Xe: Ar-Au sandwich. The vacuum level was determined from the lowenergy onset of EDC's of the gold substrate, taken before depositing the rare gas. After preparation of the thin films this onset is the same for electrons excited in the sample $(\hbar \omega=16 \mathrm{eV})$ as well as for hot electrons from the Au substrate $(\hbar \omega=19$

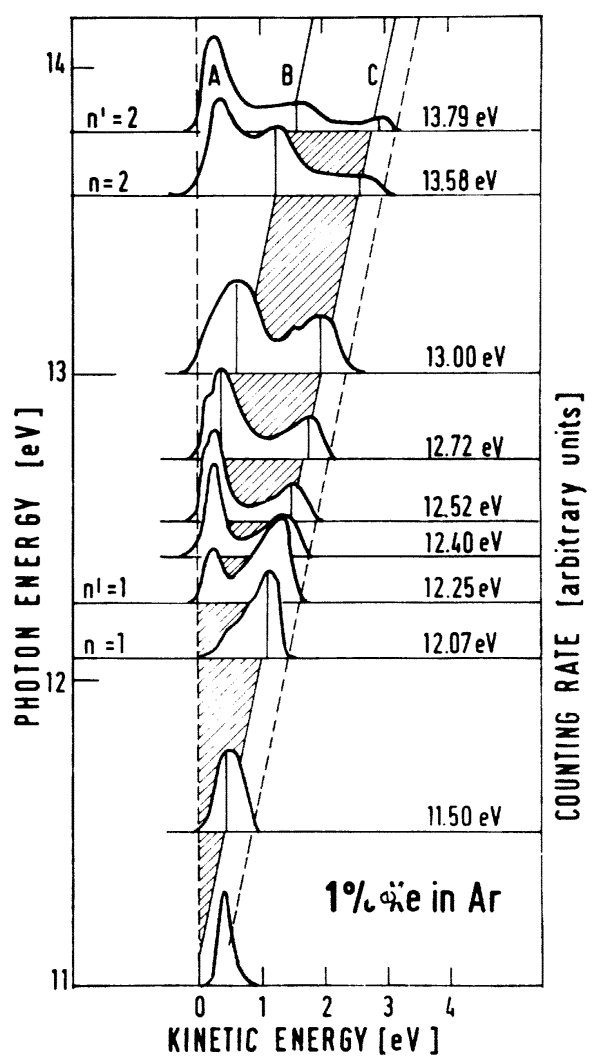

FIG. 4. Photoelectron EDC's of a 50- $\AA$-thick film of 1-at.\% Xe in Ar, similar to Fig. 3. For $A, B$, and $C$ see text.
$\mathrm{eV}$ ), indicating no change of the Au work function on evaporation (see also the left edge of the EDC's in Fig. 5).

In Figs. 3 and 4 the baselines of the spectra are shifted upwards according to the exciting energy. The kinectic energies $E_{\mathrm{kin}}$ of the maxima and onsets increase in proportion to the photon energy $\hbar \omega$ as is expected from the relation

$$
E_{\mathrm{kin}}=\hbar \omega-E_{\mathrm{th}}
$$

and demonstrated by the diagonal lines. Here $E_{\mathrm{th}}$ (threshold energy) is the binding energy of the initial state measured relative to the vacuum level $E_{V}$.

With photon energies from 11 up to $19 \mathrm{eV}$, the three different regions of excitation are covered:

(i) At low photon energies ( $\hbar \omega \leq 12 \mathrm{eV})$ the Ar host matrix is transparent. Only guest atoms are excited.

(ii) In the region of host exciton excitation $(12 \leq \hbar \omega \leq 14 \mathrm{eV})$ efficient energy transfer to the guest atoms takes place, as will be discussed in Sec. V. For this region more EDC's with an expanded photon energy scale are shown in Fig. 4.

(iii) For photon energies above $14.2 \mathrm{eV}$, the gap energy $E_{G}^{A r}$ of the Ar matrix, electrons from the guest atoms as well as from the valence band of the host are excited into the conduction bands of the host.

The strong increase of the counting rate and the change in shape for $\mathrm{Xe}$ in Ar relative to the gold substrate is demonstrated for $n=1, \hbar \omega=12.25 \mathrm{eV}$ in Fig. 5. Pure Ar of $45-\AA$ thickness yields a comparatively small increase in counting rate and an EDC peaking near zero kinetic energy, a spectrum rather similar to that of $\mathrm{Au}$. For the spectra shown in Fig. 6, the energy of transmission of the electron energy analyzer was fixed to $E_{\mathrm{kin}}=0.2$ $\pm 0.2 \mathrm{eV}$ and the photon energy was scanned (constant final-state spectra). The crosses represent the corresponding counting rates of the EDC's of the 1-at.\% Xe in Ar matrix from Fig. 4 normalized to the incident-light intensity. For the discussion it is important to keep the following observations in mind:

(a) There is a definite increase in counting rate for the Xe in Ar sample at electron energies near the vacuum level both when the $n=1$ and the $n=2$ excitons are excited.

(b) For thicker films of Xe in Ar (not shown), the counting rate in the excitonic region increases (see also Ref. 12).

(c) The shape of EDC's for pure Ar is clearly different from curves of the doped samples.

(d) The enhancement for pure Ar for a film of 45- $\AA$ thickness is smaller than for the doped sample, and with increasing thickness $(d=135 \AA)$, 


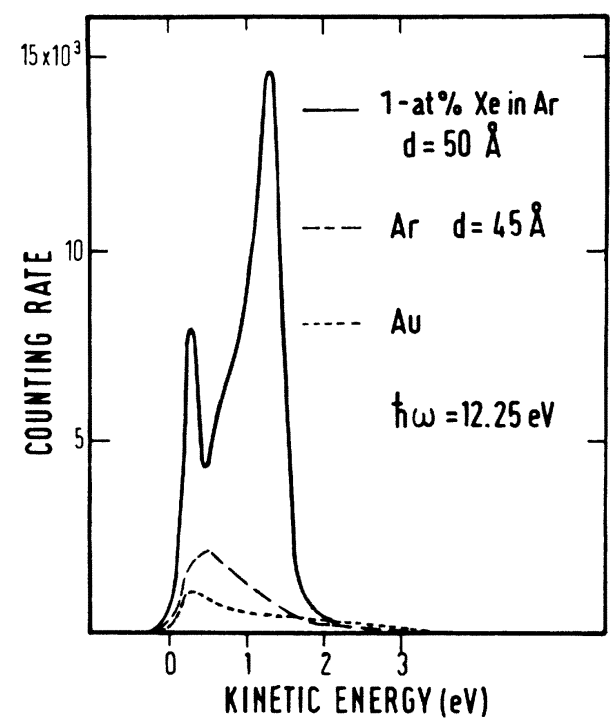

FIG. 5. Comparison of photoelectron EDC's of 1-at.\% Xe in Ar (film thickness $d=50 \AA$ ) with an EDC of pure Ar (film thickness $d=45 \AA$ ) and an EDC of the gold substrate at a photon energy of $\hbar \omega=12.25 \mathrm{eV}$. Note that the counting rates for the three curves are on the same scale.

the counting rate in the excitonic region decreases, especially in the center of the $n=1$ exciton band, and stays constant for the $n=2$ excitons (Fig. 6).

The binding energies of the initial states can be derived from the EDC at $\hbar \omega=19 \mathrm{eV}$ (Fig. 3). The variation of the photon energies (see diagonal lines) makes possible an increase in accuracy by averag-

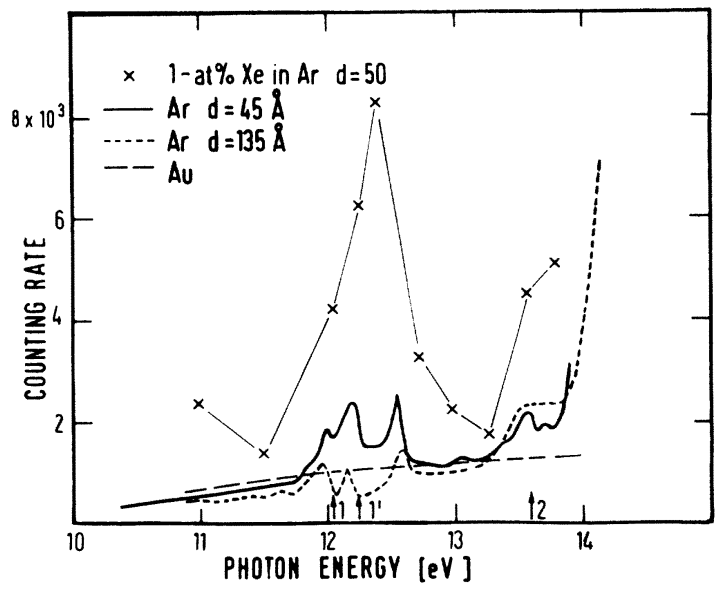

FIG. 6. Constant final state (CFS) spectra for $E_{\text {kin }}=0.2$ $\pm 0.2 \mathrm{eV}$ (counting rates at a fixed kinetic energy of the photoelectron versus the exciting photon energy) for Xe-doped Ar, for pure Ar with different film thicknesses $d$, and for the gold substrate. As a guideline the data points for Xe in Ar have been connected by a broken line (not a measured curve). ing. Furthermore shifts due to possible strong structure in the final states are not observed.

At $\hbar \omega=19$ and $16 \mathrm{eV}$ most electrons stem from the Ar $3 p$ valence bands. They produce the large peak in the left shadowed region (Fig. 3). From the kinetic energy of the high-energy onset we obtain [Eq. (1)] a binding energy for electrons at the top of the Ar $3 p$ valence bands of $E_{\mathrm{th}}^{\mathrm{Ar}}=13.9 \mathrm{eV}$. The shadowed region indicates the width of the Ar $3 p$ valence bands, $E_{\mathrm{VBW}}^{\mathrm{Ar}}$, of $1.8 \pm 0.2 \mathrm{eV}$. Owing to the smaller ionization energy, the Xe $5 p$ guest levels are located within the Ar band gap. The maximum with the highest kinetic energy corresponds to the Xe $5 p_{3 / 2}$ level, the second to the $\mathrm{Xe} 5 p_{1 / 2}$ level. The centers of the peaks are connected with diagonal lines. Their separation gives a spin-orbit splitting of the Xe $5 p$ states in an Ar matrix of $1.3 \pm 0.1 \mathrm{eV}$. The high-energy onset of the Xe excitations (dashed line) shows that the $\mathrm{Xe}$ $5 p_{3 / 2}$ level lies $3.5 \mathrm{eV}$ above the top of the Ar valence bands: $E_{\mathrm{th}}^{\mathrm{Ar}}-E_{\mathrm{th}}^{\mathrm{Xe}}$ in $\mathrm{Ar}=3.5 \mathrm{eV}$, yielding a binding energy of the Xe $5 p_{3 / 2}$ level, $E_{\mathrm{th}}^{\mathrm{Xe}}$ in $\mathrm{Ar}$, of $10.4 \pm 0.2 \mathrm{eV}$. Together with the spectroscopically determined gap values ${ }^{2,27}$ of $E_{G}^{\mathrm{Ar}}=14.15 \mathrm{eV}$ and $E_{G}^{\mathrm{Xe}}$ in $\mathrm{Ar}=10.54 \mathrm{eV}$ we get for the electron affinity, $V_{0}$, a value of $V_{0}=0.14 \pm 0.2 \mathrm{eV}$, where

$$
V_{0}=E_{G}-E_{\text {th }} \text {. }
$$

$V_{0}$ is the difference between the bottom of the conduction band and the vacuum level. All these values are compiled in Table I. As far as solid Ar is concerned they corroborate within the experimental limits the values reported earlier. ${ }^{14}$ The width of the Xe $5 p$ excitations may be partly due to the interaction of neighboring guest atoms. A close proximity is likely at a doping concentration of 1 at.\%. We have investigated films with thicknesses up to several hundred angstroms. Aside from an increase in counting rates caused by stronger absorption in the film, the main features remain and thus the given quantities are bulk properties. Charging in thicker films because of the greater separation of the positive charges from the gold substrate is stronger. The whole EDC shifts to lower kinetic energies but it can still be observed because of the preaccelerating voltage $V_{p}$. Additionally, the EDC's are broadened by the non-uniform distribution of the charges across the film. From the observed time and thickness dependence, we conclude that charging was smaller than $0.2 \mathrm{eV}$ for the spectra below $\hbar \omega=13 \mathrm{eV}$, which were measured first, and smaller than $0.3 \mathrm{eV}$ for the others.

The weak maximum with the long tail near zero kinetic energy in the spectrum for $\hbar \omega=19 \mathrm{eV}$ (Fig. 3) is caused by hot electrons from the gold substrate. From its area we get a rough estimate 
TABLE I. Parameters for the band structure and exciton states of rare-gas solids as deduced from optical (Refs. 2 and 31), photoemission yield (Refs. 12, 17, and 19) and photoelectron energy-distribution measurements (Ref. 14) together with results obtained in the present study (marked with an asterisk). All energies are in eV. $E_{\text {th }}$, threshold (binding) energies of occupied states with respect to the vacuum level; $V_{0}$, electron affinity; $E_{G}$, bandgap energy; $E(n=1)$, excitation energy, and $B(n=1)=E_{G}-E(n=1)$, binding energy of the $n=1$ exciton state; $B^{\prime}(n=1)$, binding energy calculated from the Wannier model; $\Delta E_{c}=B^{\prime}-B$, central-cell correction; SO, spin orbit.

\begin{tabular}{lccccc}
\hline \hline & $\mathrm{Ne}$ & $\mathrm{Ar}$ & $\mathrm{Xe}$ & $\mathrm{Xe}$ in Ne & Xe in Ar \\
\hline$E_{\text {th }}$ & 20.3 & 13.9 & 9.8 & $11.2^{*}$ & $10.4^{*}$ \\
$V_{0}$ & 1.4 & 0.3 & 0.5 & $1.4^{*}$ & $0.14^{*} \pm 0.2$ \\
$E_{G}$ & 21.69 & 14.2 & 9.3 & 12.6 & 10.54 \\
$E(n=1)$ & 17.83 & 12.07 & 8.36 & 9.06 & 9.22 \\
$B(n=1)$ & 3.86 & 2.13 & 0.84 & 3.54 & 1.32 \\
$B^{\prime}(n=1)$ & 5.24 & & 1.0 & 5.28 & 2.4 \\
$\Delta E_{c}$ & & & 0.06 & $1.5^{*}$ & $1.08^{*}$ \\
SO splitting (solid) & & & & $1.25 \pm 0.1^{*}$ & $1.3 \pm 0.1^{*}$ \\
SO splitting (solid, & & & 1.37 & & \\
$\quad$ calc. Ref. 28) & & & & & \\
SO splitting (gas) & & & & & \\
\hline \hline
\end{tabular}

for the photoemission efficiency of our films. From the absorption coefficient ${ }^{2} \mu$ for Ar of $\approx 6$ $\times 10^{5} \mathrm{~cm}^{-1}$, it follows that $75 \%$ of the incident light reaches the gold substrate. The absorption of Xe atoms is negligible because of the small concentration. Provided no electrons escaping from the gold are lost in the Ar film, we calculate from the ratio of the contributions from $\mathrm{Au}$ and from $\mathrm{Ar}$ an efficiency of Ar which is 7.5 times that of $\mathrm{Au}$. This assumption is reasonable according to Ref. 12. With the efficiency of $\mathrm{Au}$ of 5 to $9 \%,{ }^{28}$ we get for Ar an efficiency of 0.5 electrons emitted per photon absorbed. This value is close to the experimental result of Ref. 17 but it has to be kept in mind that we have neglected any dependence on the angle of emission.

\section{B. $\mathrm{Xe}$ in $\mathrm{Ne}$}

EDC's of 1-at.\% Xe in Ne for excitation energies below the host absorption edge $(\hbar \omega=16 \mathrm{eV})$, in the $n=1$ exciton band ( $\hbar \omega=17.5 \mathrm{eV})$ and in the $n=2$ exciton band $(\hbar \omega=20.4 \mathrm{eV})$, are presented in Fig. 7 . A structureless background due to hot electrons from the gold substrate was subtracted. The counting rates for the spectra cannot be compared, since they are arbitrarily normalized. Again the baselines of the spectra are shifted according to the photon energy and the solid diagonal line represents the expected increase of electron kinetic energy with photon energy [Eq. (1)]. Obviously, when $n=2$ excitons of the Ne host are excited, Eq. (1) does not hold and a considerable amount of energy, more than $3 \mathrm{eV}$, is missing. This striking observation, which differs from what was observed for $\mathrm{Xe}$ in $\mathrm{Ar}$, is discussed in Sec. $\mathrm{V}$ in view of the relation between energy transfer and relaxation.

The two maxima observed represent the Xe $5 p_{3 / 2}$ and $\mathrm{Xe} 5 p_{1 / 2}$ states in the band gap of the Ne matrix. Their separation gives a spin-orbit splitting of $1.25 \pm 0.1 \mathrm{eV}$ for the Xe atoms in an Ne matrix. At $\hbar \omega=16 \mathrm{eV}$, only the Xe guest atoms can be ex-

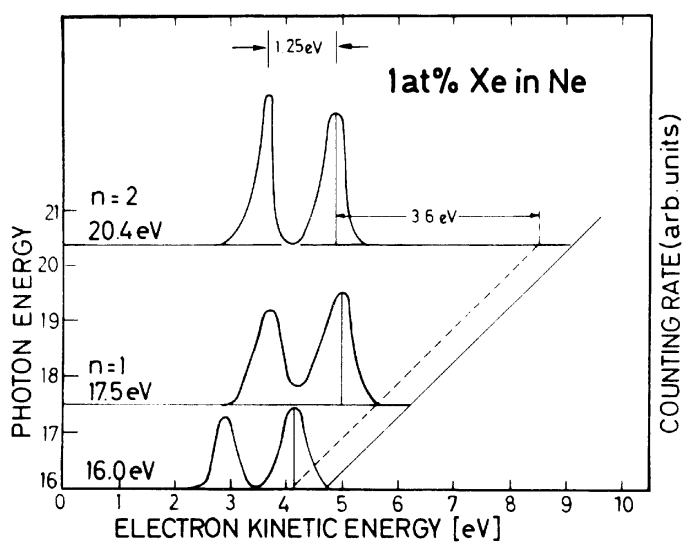

FIG. 7. Photoelectron EDC's of a thin film of Xedoped Ne for three excitation energies. The spin-orbit splitting of the Xe guest levels and the relaxation energy before energy transfer are indicated. 
cited and we do not expect relaxation processes to affect the kinetic energy of the electrons. The energy of tile high-energy onset $(4.8 \mathrm{eV})$ of the $\frac{3}{2}$ level leads to a threshold energy $E_{\mathrm{th}}^{\mathrm{Xe}}$ in $\mathrm{Ne}$ of $\mathbf{1 1 . 2}$ $\mathrm{eV}$. In the doped sample, the same EDC of the $2 p$ valence bands of $\mathrm{Ne}$ as in Ref. 14 was observed. Thus with the value of ${ }^{14} V_{0}=1.4 \mathrm{eV}$ a band gap $E_{G}^{\mathrm{Xe}}$ in $\mathrm{Ne}$ for $\mathrm{Xe}$ in $\mathrm{Ne}$ of $12.6 \pm 0.2 \mathrm{eV}$ is determined in agreement with Ref. 31 (see Table I).

\section{DISCUSSION OF THE IMPURITY STATES}

From our experiments the binding energies and the spin-orbit splitting of the Xe $5 p$ states in solid Ar and Ne matrices were determined (Table I). The spin-orbit splitting of $1.3 \mathrm{eV}$ is, within the experimental accuracy, independent of the host matrix and very close to the value in the gas phase. It also agrees with the calculated spin-orbit splitting for pure solid Xe at the center of the Brillouin zone. ${ }^{29}$ The spin-orbit splitting in EDC's of pure solid Xe is masked by the overlap of the upper and lower valence bands brought about by their $k$ dependence. Further, in optical spectra of pure Xe a well-developed exciton series of the Xe $5 p_{1 / 2}$ excitation is missing and therefore the value of 0.9 eV resulting from an extrapolation of the exciton series $^{2}$ is uncertain. The separation of $1.17 \mathrm{eV}$ observed in pure solid Xe for the $n=1$ excitons $^{2}$ may be closer to the true value for the splitting. In view of these facts the value of $1.3 \mathrm{eV}$ for the spin-orbit splitting of pure $\mathrm{Xe}$ is plausible.

From the binding energies $E_{\text {th }}^{\mathrm{Xe}}$ in $\mathrm{Ne}$ and $E_{\mathrm{th}}^{\mathrm{Xe}}$ in $\mathrm{Ar}$ together with the excitation energies of the $n=1$ excitons, the position of the later below the vacuum level can be calculated. Using the electron affinity
$V_{0}$, the exciton binding energy $B$ (the difference of excitation energy and bottom of the conduction band) is determined. Both the spin-orbit splitting and the binding energy $B$ contradict the identification of the exciton series of $\mathrm{Xe}$ in Ne given by Baldini. ${ }^{30}$ The results here are in excellent agreement, however, with the new values and assignments of Pudewill et al. ${ }^{31}$ For Xe in Ar our analysis is in accordance with Baldini. ${ }^{27}$ The present investigation of the EDC's confirms the interpretation of the optical spectra in terms of Wannier series. The discrepancy of our $B$ value with that of Gedanken et al. ${ }^{32}$ has consequences for the central-cell correction for the $n=1$ excitons. According to the calculation of Hermanson ${ }^{33}$ the central-cell correction for a specific guest atom in different matrices depends only on the binding energy $B$. With the new $B$ value for $\mathrm{Xe}$ in $\mathrm{Ne}$, the linear dependence of $B$ stated by Gedanken $e t$ al . does not hold, as is discussed in Ref. 31.

\section{ENERGY TRANSFER AND RELAXATION}

In $\mathrm{Ar}$ and Ne matrices an efficient transfer of the host exciton energy to the Xe guest atoms is observed. The main difference, documented in Figs. 4 and 7 , is the increase of the energy of the emitted electrons proportional to the excitation energy in Ar, whereas it stays constant for the $n=1$ and $n=2$ excitons in Ne. Furthermore the efficiency of the energy-transfer process for Xe in Ar is strongly dependent on the excitation energy, i.e., whether interband or exciton states are excited in the primary absorption process. In the following these observations will be discussed; see also Fig. 1(b), and Tables II and III.

TABLE II. Time hierarchy for decay processes in Xe-doped Ar and Ne. The time constants used describe the following processes: $\tau_{D}$, radiative decay; $\tau_{R}^{t}$, relaxation to trapped excitons; $\tau_{R}\left(1^{\prime}, 2 \rightarrow 1\right)$, relaxation of the $n=1^{\prime}, 2$ to $n=1$ state; $\tau_{T}$, energy transfer to Xe guest atoms; $\tau_{\text {ph }}$, relaxation to the phonon-dressed free-exciton state. Experimental results for the time hierarchy from the EDC's.

\begin{tabular}{|c|c|c|}
\hline System & $\begin{array}{l}\text { Energy } \\
\text { transfer }\end{array}$ & Time constants \\
\hline 1 -at. $\%$ Xe in Ar $n=1$ & + & $\tau_{D}>\tau_{R}^{t}>\tau_{T}$ \\
\hline$n=1^{\prime}$ & + & $\tau_{R}\left(1^{\prime} \rightarrow 1\right)>\tau_{T}$ \\
\hline$n=2$ & + & $\tau_{D}>\tau_{R}(2 \rightarrow 1)>\tau_{T} \geq \tau_{R}^{t}$ \\
\hline$\hbar \omega>E_{G}$ & - & \\
\hline 1 -at. $\% \mathrm{Xe}$ in Ne $n=1$ & + & $\tau_{D}>\tau_{T}$ \\
\hline$n=2$ & + & $\tau_{D}>\tau_{T}>\left\{\begin{array}{l}\tau_{R}(2 \rightarrow 1) \\
\tau_{\mathrm{ph}}\end{array}\right.$ \\
\hline
\end{tabular}


TABLE III. Time hierarchy from the EDC's together with theoretical calculations and estimates of the radiative decay time constants. (See Table II for definitions of time constants.) All times are in sec. For references see text.

\begin{aligned} & \hline \multicolumn{1}{c}{ System } \multicolumn{1}{c}{ Time constants } \\ & \hline 1 -at.\% Xe in Ar $n=1 \tau_{D} \approx 10^{-9}>\tau_{R}^{t} \approx 10^{-12}>\tau_{T} \\ & n=2 \tau_{D} \approx 10^{-9}>\tau_{R}\left(2 \rightarrow 1^{\prime}\right) \approx 10^{-11}>\tau_{T} \geq \tau_{R}^{t} \approx 10^{-12} \\ & 1$-at.\% Xe in Ne $n=1 \tau_{R}^{t}>\tau_{D} \approx 10^{-9}>\tau_{T} \approx 10^{-12} \\ & n=2 \tau_{R}^{t}>\tau_{D} \approx 10^{-9}>\tau_{T} \approx 10^{-12}>\left\{\begin{array}{l}\tau_{R}(2 \rightarrow 1) \approx 5 \times 10^{-13} \\ \tau_{\mathrm{ph}}\end{array}\right. \\ &$\hline \hline\end{aligned}

\section{A. $\mathrm{Xe}$ in $\mathrm{Ar}$}

\section{Interband transitions of the Ar host}

The photon energy $\hbar \omega=19 \mathrm{eV}$ is sufficient to excite electrons from the whole Ar valence bands into the Ar conduction bands (Fig. 3). Previous yield measurements of pure Ar showed an efficiency of 0.6 electrons per photon absorbed. ${ }^{17}$ Yield spectra of thin films of Xe-doped Ar indicated a comparable efficiency, ${ }^{12}$ but it could not be decided whether the electrons were emitted by the host or the guest atoms. The different origins of the electrons are clearly separated in the EDC's in Fig. 3. The contribution of electrons from the Ar $3 p$ valence bands is 80 times that of Xe $5 p$ states at $16 \mathrm{eV}$ and 40 times at $19 \mathrm{eV}$. This result, namely, that the electrons are emitted with a probability roughly according to the atomic concentrations, leads to the conclusion that they stem from distinct photon absorption processes at Ar or Xe atoms, respectively. The deviations from the given mixing concentrations may be due to different absorption coefficients of guest and host atoms and to an enrichment of Xe in the Ar matrix during solidification. An enrichment factor of 2 (or of 3 when taking into account the energy dependence of the Ar absorption constant) is in agreement with the observation of Baldini. ${ }^{30}$ In any case we can give an upper limit of 0.01 for the efficiency of the energy-transfer process to $\mathrm{Xe}$ atoms both for excitation energies of 16 and $19 \mathrm{eV}$. We conclude that for the Ar interband transitions no energy transfer, or at most with an efficiency below 0.01 , takes place in photoemission.

This observation does not exclude the possibility that in luminescence experiments energy transfer will be seen when electrons are excited into the conduction band. As stated before, luminescence is a much slower process than photoelectron emission, since for the light emission the hole has to capture an electron again. Before light is emitted the captured electron may relax from the conduction band to exciton states (see calculation of Ref. 11). Finally energy transfer may take place from the host exciton states to the Xe guest, a process discussed in Sec. VA2. Alternatively the hole in the Ar valence band may be filled by an electron from the Xe guest atoms instead of a free electron. The resulting hole at the Xe guest atom may subsequently capture an electron, leading to radiative decay. The observed photon energy will be equal to that from the energy-transfer processes.

\section{Ar excitonic region}

An increase of electron emission from the Xe guest atoms of 2 orders of magnitude is observed in the Ar-host exciton regime. According to the Ar absorption constant ${ }^{26}$ in the $n=1$ exciton $80 \%$ of the light will excite Ar excitons and only $0.003 \%$ should ionize the Xe guest atoms directly for the film thickness of $50 \AA$. The $n=1$ and $n=2$ excitons lie below the Ar vacuum level. They can contribute to photoemission only by secondary processes. (This is evident from an extrapolation of the kinetic energy of the Ar valence-band excitations in Fig. 3.) Now let us consider the various possible processes. Energy transfer to the gold substrate yields only a small contribution. As observed for pure ${ }^{17} \mathrm{Ar}$ and studied for pure ${ }^{34} \mathrm{Xe}$ it has a much lower efficiency and a different dependence on thickness (see Figs. 5 and 6, and Ref. 17) than observed for Xe in Ar. Further, the shape of the EDC from pure $\mathrm{Ar}$ at $\hbar \omega=12.25 \mathrm{eV}$ is similar to that of $\mathrm{Au}$ and does not correspond to that of $\mathrm{Xe}$ in Ar (Fig. 5). We conclude that almost all of the emitted electrons in the excitonic region are produced by an energy-transfer process to the Xe atoms. There the yield almost reaches the same value as in the maximum of host emission in the interband regime at $\hbar \omega \approx 15 \mathrm{eV}$.

In Ref. 12 the dependence of the total yield on film thickness and Xe concentration was studied and interpreted in terms of a diffusion model. The 
EDC's give more detailed insight in the transfer process.

Energy transfer from the $n=1$ excitons. For photon energies of 11 and $11.5 \mathrm{eV}$ the Ar matrix is transparent and the Xe atoms are excited directly. No deviations from the straight connections of onsets and peak positions in the EDC's from direct excitations at $\hbar \omega=11$ and $11.5 \mathrm{eV}$ with those at $\hbar \omega=16$ and $19 \mathrm{eV}$ are observed for $\hbar \omega=12.07$ and $12.25 \mathrm{eV}$. Thus we have to exclude dissipation of energy by relaxation prior to energy transfer from the $n=1$ and $n^{\prime}=1$ exciton states $\left(n^{\prime}\right.$ denotes the spin-orbit partner to $n$ ) to the Xe guest, at least within the experimental limit of $0.2 \mathrm{eV}$.

On the basis of luminescence spectra resonant energy transfer from the self-trapped matrix excitons to the guest atoms was assumed in Ref. 35 . Generally in luminescence experiments of pure solid Ar emission from the self-trapped excitons is observed (Fig. 8). The strongest emission band found in all investigations is centered at $9.8 \mathrm{eV}$. Its intensity falls below $10 \%$ within $\pm 0.5 \mathrm{eV}$ (Fig. 8). Therefore even the high-energy part of this molecular emission band is not sufficient to ionize the Xe atoms with the observed efficiency. (Note that the ionization energy for Xe atoms in an Ar matrix is $10.4 \mathrm{eV}$.) Furthermore the energy of the selftrapped excitons is more than $2 \mathrm{eV}$ too low to ionize both spin-orbit-split levels (Fig. 8). Therefore we have to reject the resonant energy-transfer process from self-trapped host excitons, which was proposed in Ref. 35 as the major energytransfer mechanism. Rather, the energy of the free excitons is transferred to Xe guest atoms. In Refs. 5 and 6 weak luminescence bands at higher energies have been detected under certain con-

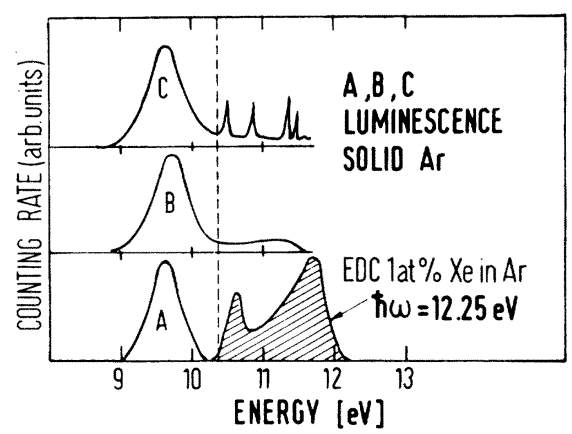

FIG. 8. Comparison of luminescence spectra of pure Ar (solid curves A, B, C are measurements from Refs. 35,5 , and 6 , respectively) with the EDC (hatched curve) of a Xe-doped Ar film. The energy scale refers to the energy of the emitted photons. For the EDC it denotes the energy of the photoelectrons relative to the top of the Xe guest levels. The dashed vertical line marks the vacuum level, denoting the minimum energy required for ionization of the Xe impurity levels. ditions (Fig. 8). It is still an open question if they belong partly to emission from free excitons. Again the intensity and the energies are too small to explain the EDC.

The difference in the shape of the EDC for excitation of the $n\left(\frac{3}{2}\right)=1$ at $12.07 \mathrm{eV}$ and of the $n^{\prime}\left(\frac{1}{2}\right)$ $=1$ at $12.25 \mathrm{eV}$ (Fig. 4) can be attributed to larger values of the transferred energy, which allows also ionization of the lower Xe $5 p_{1 / 2}$ level. This difference demonstrates that relaxation of the $n^{\prime}=1 \rightarrow n=1$ needs more time than energy transfer. Summarizing the observations for the $n=1$ and $n^{\prime}=1$ exciton we obtain for $1-$ at. $\% \mathrm{Xe}$ in $\mathrm{Ar}$ :

(i) Energy transfer (time constant $\tau_{T}$ ) is faster radiative decay $\left(\tau_{D}\right): \tau_{T}<\tau_{D}$.

(ii) $\tau_{T}$ is smaller than the relaxation time constant $\tau_{R}^{t}$ to bound states (trapped exciton): $\tau_{T}<\tau_{R}^{t}$.

(iii) $\tau_{T}$ is smaller than the relaxation time constant $\tau_{R}\left(n=1 \rightarrow n^{\prime}=1\right)$ for relaxation of $n^{\prime}=1$ to $n=1$ exciton: $\tau_{T} \rightarrow \tau_{R}\left(n=1 \rightarrow n^{\prime}=1\right)$.

Using the oscillator strength of the $n=1$ exciton of $\mathrm{Ar},{ }^{26}$ a time constant of $\tau_{D} \approx 10^{-9} \mathrm{sec}$ is estimated for the radiative decay of these excitons. From the dominant contribution of the self-trapped excitons to luminescence of pure Ar a ratio of $\tau_{R}^{t} / \tau_{D} \lessgtr \frac{1}{100}$ was estimated by Gedanken et al. ${ }^{35}$ resulting in an upper limit for $\tau_{R}^{t}$ of $10^{-11} \mathrm{sec}$. This agrees with a calculation of Martin, ${ }^{10}$ which predicts a dissipation of energy of 0.5 to $1 \mathrm{eV}$ within a time of $10^{-12}$ sec by self-trapping of the exciton in solid Ar. According to our EDC's, the relaxation energy before energy transfer is smaller than $0.2 \mathrm{eV}$ leading to the following timo hierarchy:

$$
\tau_{D} \approx 10^{-9}>\tau_{R}^{t} \approx 10^{-12}>\tau_{T}
$$

and

$$
\tau_{R}\left(n=1 \rightarrow n^{\prime}=1\right)>\tau_{T} .
$$

Energy transfer from higher excitons $n=2, n^{\prime}=2$. The shape of the EDC's changes with photon energy (Fig. 4). At higher energies a third maximum, $A$, appears near the vacuum level. At lower photon energies this maximum partly overlaps with that of the $\mathrm{Xe} 5 p_{1 / 2}$ level. But at energies corresponding to the $n=2(\hbar \omega=13.58 \mathrm{eV})$ and $n^{\prime}=2$ $(\hbar \omega=13.79 \mathrm{eV})$ exciton states the maxima $B$ and $C$ are clearly separated from maximum $A$ (Fig. 4). Thus the EDC can be separated into two parts: maxima $B$ and $C$, which are caused by electrons from an ionization process of the Xe $5 p_{3 / 2}$ and Xe $5 p_{1 / 2}$ levels, and maximum $A$ at higher energies, which possibly contributes with a flat background to $B$ and $C$.

First we conclude that the relaxations times $\tau_{R}(n=2 \rightarrow n=1)$ and $\tau_{R}\left(n=2^{\prime} \rightarrow n=1^{\prime}\right)$ for the processes $n=2 \rightarrow n=1,1^{\prime}$ and $n=2^{\prime} \rightarrow n=1,1^{\prime}$ are long 
compared to the processes leading to photoemission, because the maxima $A, B$, and $C$ do not correspond in their kinetic energies and shape to the EDC's at the excitation energies of the $n=1$ or $n^{\prime}=1$ excitons. Next we attribute the maxima $B$ and $C$ to energy transfer of the free (i.e., not relaxed) $n=2\left(n^{\prime}=2\right)$ excitons to the Xe guest atoms, because they are located at the diagonal lines [Fig. 4, Eq. (1)].

The origin of the maximum $A$ and the backgound contribution to $B$ and $C$ cause some problems. Since they appear at photon energies below $E_{\mathrm{th}}^{\mathrm{Ar}}$ they must originate from an energy-transfer process. One possibility may be energy transfer to the gold substrate as was observed for pure Ar (Fig. 6). For a 135- $\AA$-thick film of pure Ar the intensity of slow electrons is reduced in the region of the $n=1$ exciton, as we would expect from the small penetration depth of the light, with the consequence that the excitons are excited a greater distance from the substrate, leading to lower efficiency of energy transfer to the gold (see also Ref. 34). In the region of the $n=2$ and $n^{\prime}=2$ excitons of pure Ar, because of the larger penetration of the light, photoemission stays constant according to the balance of increased absorption in the film and reduced efficiency of energy transfer to the substrate. However, for the origin of the peak $A$ in the EDC's from Xe-doped Ar we rule out the energy transfer to the Au substrate:

(i) For a $45-\AA$-thick film of pure Ar there is an increase in photoemission compared to pure gold, but the increase is a factor of 3 less than the contribution of maximum $A$ for a 50 - $\AA$-thick film of 1-at.\% in Ar (see Figs. 5 and 6).

(ii) In addition the ratio of maximum $A$ to maximum $B$ or $C$ did not change markedly with film thickness. If maximum $A$ would be produced by energy transfer to the substrate, the ratio should be lowered for thicker films because the excitons are excited at a larger distance from the substrate and energy transfer to the Xe atoms (maxima $B$ and $C$ ) should be favored.

For these reasons energy transfer to the gold substrate can only be a process of minor signif icance in forming maximum $A$. We rather suggest that maximum $A$ is due to energy transfer from partly relaxed excitons. Since the major part of peak $A$ has kinetic energies lower than from the direct transfer from $n=1$ and $n=2$ states, maximum $A$ originates from energy transfer of bound exciton states.

We then would have to state that the excitation of the $n=1,1^{\prime}$ excitons leads to a direct energy transfer prior to relaxation, whereas excitation of the $n=2,2^{\prime}$ excitons partly leads to direct energy transfer (peaks $B$ and $C$ ) and partly to energy transfer from a relaxed bound exciton state (peak $A$ ). This different behavior of the $n=1$ and $n=2$ excitons can be attributed to a lower-energy transfer rate or a faster trapping time $\tau_{R}^{t}$ of the $n=2$ excitons.

After relaxation by $\approx 0.3 \mathrm{eV}$ the energy above the trapping minimum is dissipated very fast, according to the calculation of Martin. ${ }^{10}$ Because we observe in the EDC of the $n=2$ excitons also appreciable energy transfer of free excitons, the time constant for energy transfer, $\tau_{T}$, may be only slightly larger than the trapping time $\tau_{R}^{t}$. Radiative decay $\left(\tau_{D}\right)$ has only been detected from states lower than $n=2$. Thus the following time hierarchy is obtained:

$$
\tau_{D}>\tau_{R}(n=2-n=1)>\tau_{T} \gtrless \tau_{R}^{t} .
$$

With our estimated $\tau_{D}$ and $\tau_{R}^{t}$ from the calculation of Martin we get upper and lower limits for $\tau_{R}(n=2-n=1): 10^{-9}>\tau_{R}(n=2 \rightarrow n=1)>10^{-12} \mathrm{sec}$. Webman et al. ${ }^{11}$ deduced from a model calculation a relaxation time constant for an impurity state in solid Ar: $\tau_{R}\left(n=2 \rightarrow n^{\prime}=1\right) \approx 10^{-11} \mathrm{sec}$, a result compatible with the limits obtained above. Taking these numbers leads to

$$
\begin{aligned}
\tau_{D} & \approx 10^{-9}>\tau_{R}\left(n=2 \rightarrow n^{\prime}=1\right) \approx 10^{-11}>\tau_{T} \geq \tau_{R}^{t} \\
& \approx 10^{-12} \mathrm{sec} .
\end{aligned}
$$

The consequences of the energy dependence of the energy-transfer rate constant should be reconciled with the explanations in terms of an exciton diffusion or Förster-Dexter model, or with an integration of both. ${ }^{12,34}$ For this discussion the information from the present EDC measurements seems not to be sufficient.

We note that in Ref. 12 for Xe in Ar a rate constant for energy transfer of $S=6 \times 10^{-8} \mathrm{~cm} \mathrm{sec}^{-1}$ was determined, in particular from the line shape and the concentration dependence of the photoelectron emission yield in the $n=2$ exciton regime. This value results for 1 -at.\% Xe in Ar in an effective lifetime of $5 \times 10^{-11} \mathrm{sec}$ which is mainly determined by energy transfer. Thus, within the experimental accuracy, this value is not far from the estimated limit for $\tau_{T}$.

\section{B. $\mathrm{Xe}$ in $\mathrm{Ne}$}

For 1-at.\% Xe in $\mathrm{Ne}$ we find again an efficient energy-transfer process from the excitons of the matrix to the guest atoms. This transfer process was also found in recent photoemission yield measurements. ${ }^{19}$ The EDC's presented in Fig. 7 are evidence for this process. Both the shape and the energy of the two maxima observed are within the experimental accuracy the same for excitation energies corresponding to the 
$n=1$ and $n=2$ excitons. This behavior is very different from the results discussed above for Xedoped Ar and leads us to the following statements:

(i) The relaxation time constant $\tau_{R}(n=2 \rightarrow n=1)$ is small relative to the time constants for energy transfer $\tau_{T}$. Thus an energy of $\approx 3.6 \mathrm{eV}$ is dissipated before energy transfer takes place. We note that this is the difference of the excitation energies for the $n=1$ and $n=2$ states.

(ii) When the $n=2$ excitons are excited in the $\mathrm{Ne}$ matrix there is no indication for an additional relaxation to bound exciton states before energy transfer compared to $n=1$. This is contrary to the case of Ar, where such a process very likely caused peak $A$ (Fig. 4). For Ne the Au background (subtracted in Fig. 7) was the same for excitation into the $n=2$ and $n=1$ exciton states.

(iii) At the photon energy of $16 \mathrm{eV}$ the Ne matrix is transparent and the absorbed photons ionize the $\mathrm{Xe}$ guest atoms directly. The EDC for the $n=1$ exciton is shifted by $0.7 \pm 0.2 \mathrm{eV}$ to lower energies compared with the energy expected from Eq. (1). In Fig. 7 this fact is evident in the distance to the diagonal line. This relaxation energy corresponds to the Stokes shift of $0.9 \mathrm{eV}$ for the strong emission band observed in luminescence. ${ }^{36,37}$ As was discussed by Jortner et al. ${ }^{37}$ this luminescence is due to the radiative decay of the free but phonondressed excitons.

Thus the energy of the free $n=1$ excitons is transferred to the Xe guest atoms with the excitons in an intermediate state of phonon relaxation but without relaxation to the trapped state. The transferred energy is independent of whether the $n=1$ or $n=2$ excitons of the Ne host are excited.

From the above observations the following time hierarchy, with $\tau_{\mathrm{ph}}$ the phonon relaxation time constant and $\tau_{R}^{t}$ the time constant for relaxation to trapped excitons, is deduced:

$$
\tau_{R}^{t}>\tau_{D}>\tau_{T}>\left\{\begin{array}{l}
\tau_{R}(n=2 \rightarrow n=1) \\
\tau_{\mathrm{ph}}
\end{array} .\right.
$$

From the oscillator strength of the $n=1$ excitons from Ref. 31 a lifetime $\tau_{D} \approx 10^{-9} \mathrm{sec}$ for the radiative decay can be estimated. The rate constant $S \tau_{0}$ between $1 \leq S \tau_{0} \leq 10^{-2} \mathrm{ppm}^{-1}$ from Ref. 19 for the energy transfer of Ne exciton energy to guest atoms gives an effective exciton lifetime $\tau$ which is mainly determined by energy transfer: $10^{-13}$ $\leq \tau \leq 10^{-11}$ sec. Using these values we get (in sec)

$$
\tau_{R}^{t} \geq \tau_{D} \approx 10^{-9}>\tau_{T} \approx 10^{-12}>\tau_{R}(n=2 \rightarrow n=1) .
$$

A theoretical estimate of Webmann et al.$^{11}$ for $\tau_{R}(n=2 \rightarrow n=1)$ with $\tau_{R}(n=2 \rightarrow n=1) \approx 5 \times 10^{-13} \mathrm{sec}$ is compatible with this time hierarchy.

\section{ACKNOWLEDGMENTS}

We want to thank A. Harmsen, F.-J. Himpsel, D. Pudewill, and V. Saile for help in the experiments and valuable discussions. The support of the experiments by Dr. M. Skibowski and Professor W. Steinmann is gratefully acknowledged. The work was stimulated by the discussions with Professor Joshua Jortner.
*Work supported by Deutsche Forschungsgemeinschaft (DFG) and Bundesminsterium für Forschung and Technologie (BMFT).

${ }^{1}$ (a) J. B. Birks, Photophysics of Aromatic Molecules (Wiley, New York, 1970); (b) S. P. McGlynn, T. Azumi, and M. Kinoshita, Molecular Spectroscopy of the Triplet State (Prentice-Hall, Englewood Cliffs, N. J., 1969); (c) B. R. Henry and M. Kasha, Ann. Rev. Phys. Chem. 19, 161 (1968); (d) J. B. Birks and I. H. Munro, Prog. React. Kinet. 4 , 239 (1967); (e) J. Jortner, S. A. Rice, and R. M. Hochstrasser, Advances in Photochemistry, edited by W. A. Noyes, J. N. Pitts, and G. Hammond (Wiley, New York, 1969), Vol. 7 .

${ }^{2}$ For a recent review, see B. Sonntag, in Rare Gas Solids, edited by M. L. Klein and J. A. Venables (Academic, New York, 1975).

${ }^{3} \mathrm{~J}$. Jortner, in Vacuum Ultraviolet Radiation Physics, edited by E. E. Koch, R. Haensel, and C. Kunz (Pergamon-Vieweg, Braunschweig, 1974), p. 263.

${ }^{4}$ M. Creuzburg, Solid State Commun. $\underline{9}, 665$ (1971), and references therein.

${ }^{5}$ R. Broadmann, R. Haensel, U. Hahn, U. Nielsen, and G. Zimmerer, in Vacuum Ultraviolet Radiation
Physics, edited by E. E. Koch, R. Haensel, and C. Kunz (Pergamon-Vieweg, Braunschweig 1974), p. 344 , and references therein.

${ }^{6}$ A. Bonnot, A. M. Bonnot, R. Coletti, J. M. Debever, and J. Hanus, J. Phys. (Paris) 35, C3-49 (1974).

${ }^{7}$ (a) J. Jortner, L. Meyer, S. A. Rice, and E. G. Wilson, J. Chem. Phys. 42, 4250 (1963); (b) N. G. Basov, V. A. Danilychev, Ya.M. Popov, and D. D. Khodkevich, JETP Lett. 12, 329 (1970).

${ }^{8}$ (a) D. J. Bradley, D. R. Hull, and M. H. R. Hutchinson McGeoch, Opt. Commun. 11, 335 (1974); (b) R. W. Dreyfus and S. C. Wallace, Opt. Commun. 13, 218 (1975).

${ }^{9}$ A. Gedanken, J. Jortner, B. Raz, and A. Szöke, J. Chem. Phys. 57, 3456 (1972).

${ }^{10}$ (a) M. Martin and S. A. Rice, Chem. Phys. Lett. 7, 94 (1970); (b) M. Martin, J. Chem. Phys. 54, 3291 (1971).

${ }^{11} \mathrm{I}$. Webmann and J. Jortner (unpublished); see also Ref. 3.

${ }^{12}$ Z. Ophir, B. Raz, J. Jortner, V. Saile, N. Schwentner, E. E. Koch, M. Skibowski, and W. Steinmann, J. Chem. Phys. 62, 650 (1975).

${ }^{13}$ For example, P. M. Rentzepis, Science 163, 239 
(1970).

${ }^{14}$ N. Schwentner, F. J. Himpsel, V. Saile, M. Skibowski, W. Steinmann, and E. E. Koch, Phys. Rev. Lett. 34 , 528 (1975).

${ }^{15}$ N. Schwenter, Phys. Rev. B (to be published).

${ }^{16} \mathrm{~N}$. Schwentner, F. J. Himpsel, E. E. Koch, V. Saile, and M. Skibowski, in Vacuum Ultraviolet Radiation Physics, edited by E. E. Koch, R. Haensel, and C. Kunz (Pergamon-Vieweg, Braunschweig, 1974), p. 335.

${ }^{17}$ (a) N. Schwentner, M. Skibowski, and W. Steinmann, Phys. Rev. B $\underline{8}, 2965$ (1973); (b) E. E. Koch, B. Raz, V. Saile, N. Schwentner, M. Skibowski, and W. Steinmann, Jpn. J. Appl. Phys. Suppl. 2, Pt. 2, 775 (1974).

${ }^{18}$ E. E. Koch, V. Saile, N. Schwentner, and M. Skibowski, Chem. Phys. Lett. 28, 562 (1974).

${ }^{19}$ D. Pudewill, F. J. Himpsel, V. Saile, N. Schwentner, M. Skibowski, E. E. Koch, and J. Jortner, J. Chem. Phys. (to be published).

${ }^{20}$ E. E. Koch and M. Skibowski, Chem. Phys. Lett. $\underline{9}$, 429 (1971).

${ }^{21}$ U. Backhaus, Diplomarbeit (Universität Hamburg, 1973) (Int. Rep. DESY F41-73/11) (unpublished).

${ }^{22}$ N. Schwentner, E. E. Koch, V. Saile, M. Skibowski, and A. Harmsen, in Vacuum Ultraviolet Radiation Physics, edited by E. E. Koch, R. Haensel, and C. Kunz (Pergamon-Vieweg, Braunschweig, 1974), p. 792

${ }^{23} \mathrm{~J}$. F. O'Brien and K. J. Teegarden, Phys. Rev. Lett 17 , 919 (1966).
${ }^{24}$ S. V. Pepper, J. Opt. Soc. Am. 60, 805 (1970).

${ }^{25} \mathrm{~K}$. Platzöder and W. Steinmann, J. Opt. Soc. Am. 58 , 588 (1968).

${ }^{26}$ A. Harmsen, E. E. Koch, V. Saile, N. Schwentner, and M. Skibowski, in Vacuum Ultraviolet Radiation Physics, edited by E. E. Koch, R. Haensel, and C. Kunz (Pergamon-Vieweg, Braunschweig, 1974), p. 339.

${ }^{27}$ G. Baldini and R. S. Knox, Phys. Rev. Lett. 11,127 (1963).

${ }^{28}$ R. B. Cairns and J. A. R. Samson, J. Opt. Soc. Am. $\underline{56}$, 1568 (1966).

${ }^{29}$ U. Rössler, Phys. Status Solidi B 42,345 (1970).

${ }^{30}$ G. Baldini, Phys. Rev. 137, A508 (1965).

${ }^{31}$ D. Pudewill, F. J. Himpsel, V. Saile, N. Schwentner, M. Skibowski, and E. E. Koch, Phys. Status Solidi B 74, 485 (1976).

${ }^{32}$ A. Gedanken, B. Raz, and J. Jortner, J. Chem. Phys. 58,1178 (1973).

${ }^{33}$ J. Hermanson, Phys. Rev. 150, 150 (1966).

${ }^{34} \mathrm{Z}$. Ophir, N. Schwentner, B. Raz, M. Skibowski, and J. Jortner, J. Chem. Phys. 63,1072 (1975).

${ }^{35}$ A. Gedanken, B. Raz, and J. Jortner, J. Chem. Phys. 59, 5471 (1973).

${ }^{36}$ See, for example, R. E. Packard, F. Reif, and C. M. Surko, Phys. Rev. Lett. 25, 4628 (1972); I. Ya. Fugol, E. V. Sarchenko, and A. G. Belov, JETP Lett. 16, 172 (1972).

${ }^{37}$ A. Gedanken, B. Raz, and J. Jortner, J. Chem. Phys. 59, $1630(1973)$. 